Research Paper

\title{
Exosomes Derived from Hypoxic Colorectal Cancer Cells Transfer Wnt4 to Normoxic Cells to Elicit a Prometastatic Phenotype
}

\author{
Zhe Huang, Mingli Yang, Yunze Li, Fan Yang, Yong Feng ${ }^{\bowtie}$ \\ Department of $11^{\text {th }}$ general surgery, Shengjing Hospital of China medical university, Shenyang, China. \\ $\triangle$ Corresponding author: Yong Feng, Department of 11th general surgery, Shengjing Hospital of China medical university, Shenyang, China. TEL: \\ 00-86-024-96615; Email: fengy@sj-hospital.org \\ (1) Ivyspring International Publisher. This is an open access article distributed under the terms of the Creative Commons Attribution (CC BY-NC) license \\ (https://creativecommons.org/licenses/by-nc/4.0/). See http://ivyspring.com/terms for full terms and conditions.
}

Received: 2018.07.03; Accepted: 2018.09.28; Published: 2018.11.13

\begin{abstract}
Hypoxia is the most common characteristic of solid tumours driving cancer metastasis. Cancer cells release exosomes with various functions into the tumour microenvironment during cancer progression. However, the roles and associated mechanisms of hypoxic colorectal cancer (CRC) cell-derived exosomes remain poorly understood. Here, we found that exosomes secreted by hypoxic CRC cells promoted the migration and invasion abilities of normoxic CRC cells. Inhibition of exosome secretion by GW4869 reduced hypoxic exosome-mediated migration and invasion of normoxic CRC cells. Furthermore, we found that these hypoxic exosomes contained Wnt4 depending on HIFla. Exosomal Wnt4 mediated hypoxic exosome-mediated migration and invasion of normoxic CRC cells. Moreover, exosomal Wnt4 enhanced $\beta$-catenin translocation to the nucleus in normoxic CRC cells. The activation of $\beta$-catenin signalling was important for the migration and invasion of normoxic CRC cells, which was eliminated by treatment with the $\beta$-catenin inhibitor ICG-001. Taken together, the results of our study indicate that hypoxia may stimulate tumour cells to release Wnt4-rich exosomes that are delivered to normoxic cells to enhance prometastatic behaviours, which might provide new targets for CRC treatment.
\end{abstract}

Key words: colorectal cancer, hypoxia, exosome, prometastatic, Wnt4, $\beta$-catenin

\section{Introduction}

Colorectal cancer (CRC), especially metastatic $\mathrm{CRC}$, is one of the most common causes of cancer-related death and has therefore attracted much attention from researchers for many years (1-2). Approximately $50-60 \%$ of patients with CRC present with metastases at initial diagnosis (3). Because metastasis is the leading cause of CRC treatment failure, there is an imperative need to elucidate the molecular mechanisms driving this process (4). A hypoxic tumour microenvironment, which is defined as a condition in which the oxygen pressure in the tumour tissue is less than 5 to $10 \mathrm{~mm} \mathrm{Hg}$, is extremely important for cancer metastasis $(5,6)$. Hypoxiainducible factors (HIFs), especially HIF-1a, are largely responsible for mediating adaptive reactions to hypoxia (6).
Exosomes are nano-sized membrane vesicles with diameters between 30-100 $\mathrm{nm}$ and are generated from endosomal compartment invaginations (7-9). As previously reported, colorectal cancer cell-derived exosomes have important roles in tumour progression including invasion, angiogenesis, immune modulation and distal metastasis through effectively delivering microRNAs, mRNAs and proteins (10-12). We previously found that exosomes released from hypoxic CRC cells enhanced tumour growth and angiogenesis by enhancing the proliferation and migration of endothelial cells (13). However, the functions and underlying molecular mechanisms of hypoxic CRC cell-derived exosomes are still largely unknown. 
Wnt/ $\beta$-catenin signalling directs crucial physiological and pathological processes during metazoan development and is abnormally triggered in cancers including CRC (14-16). Wnt4 is a member of the Wnt family that has been shown to participate in carcinogenesis (17-19). Wnt4 promotes the proliferation of cancer stem cells in response to progesterone in breast cancer (20). The upregulation of Wnt4 has also been detected in gastric cancer (21). Consistent with these findings, we found that Wnt 4 was enriched in exosomes released from hypoxic CRC cells and mediated the functions of endothelial cells (13).

In this study, we sought to identify new functions of hypoxic CRC cell-derived exosomes. We found that exosomes released from hypoxic CRC cells enhanced the migration and invasion abilities of normoxic CRC cells. Further, hypoxic exosomal Wnt4 mediated hypoxic exosome-mediated migration and invasion of normoxic CRC cells. Exosomal Wnt4 enhanced nuclear translocation of $\beta$-catenin in normoxic CRC cells. Stimulation of $\beta$-catenin signalling was important for the migration and invasion of normoxic CRC cells and could be reduced via $\beta$-catenin inhibitor ICG-001. In conclusion, our study suggests that hypoxia may stimulate tumour cells to release Wnt4-rich exosomes that are then endocytosed by normoxic cells to promote metastasis. Importantly, this study may provide new targets for CRC treatment, especially treatment of metastatic CRC.

\section{Materials and Methods}

\section{Cell culture}

The human CRC cell lines HT29 and HCT116 were purchased from the Stem Cell Bank of the Chinese Academy of Sciences. HT29 and HCT116 cells were maintained in RPMI-1640 supplemented with $10 \%$ exosome-depleted foetal bovine serum (FBS; Gibco, Carlsbad, CA, USA), penicillin (100 units/mL), and streptomycin $(100 \mu \mathrm{g} / \mathrm{mL})$ at $37^{\circ} \mathrm{C}$ in a humidified atmosphere containing $5 \% \mathrm{CO}_{2}$. All cells were verified to be free of mycoplasma contamination.

\section{Exosome isolation}

To isolate exosomes, the CRC cell lines HT29 and HCT116 were treated with $250 \mu \mathrm{M} \mathrm{CoCl}_{2}$ (22) for $48 \mathrm{~h}$, while the normoxic cells were cultured without $\mathrm{CoCl}_{2}$ treatment. We then centrifuged the supernatants twice $(1000 \mathrm{~g} \times 10 \mathrm{~min}, 3000 \mathrm{~g} \times 30 \mathrm{~min})$ to remove cells or cell fragments, treated them with a total exosome isolation kit (Life Technology) overnight, and then centrifuged them again $(10000 \mathrm{~g} \times 1 \mathrm{~h})$. Isolated exosomes were re-suspended in PBS and stored at $-80^{\circ} \mathrm{C}$. The concentration of exosomal protein was determined by a BCA Assay.

\section{Western blot}

To determine the expression of the exosomal marker CD63, Western blotting was performed with the following antibodies: rabbit anti-human CD63 (ab59479, Abcam; 1:1000) and mouse anti-actin (Millipore; 1:10,000). Briefly, samples were lysed with lysis buffer (50 mM Tris- $\mathrm{HCl}$ (pH 7.5), $150 \mathrm{mM} \mathrm{NaCl}$, and $1 \%$ Triton X-100) containing protease inhibitors. In total, $30 \mu \mathrm{g}$ lysate was loaded on 10\% SDS-PAGE gels and transferred to PVDF membranes. The membranes were incubated with primary antibodies overnight at $4{ }^{\circ} \mathrm{C}$, followed by incubation with an HRP-conjugated secondary antibody. The bound antibodies were detected using an ECL kit (Millipore, WBKLS0500).

\section{Lentiviral vector-mediated HIF $1 \alpha$ or Wnt4 knockdown}

The target sequences for HIF1a knockdown were SH1: 5'-CAGAAATGGCCTTGTGAAA-3'; SH2: 5'-GATGGAAGCACTAGACAAA-3'; SH3: 5'-CCTA ATAGTCCCAGTGAAT-3'; and SH4: 5' -AGGAAGAA CTAAATCCAAA- $3^{\prime}$. The target sequences for Wnt4 knockdown were SH1: 5' - GGTCAGGATGCTCTGAC AACA-3'; SH2: 5'-GGCCTTCTCACAGTCGTTTGT -3'; SH3: 5'-GCTCCTGTGAGGTAAAGACGT-3'; and SH4: 5'- GTCAGGCTCCTGTGAGGTAAA-3'. After $48 \mathrm{~h}$, the knockdown efficiency was examined by qRT-PCR and Western blot.

Lentiviral vectors and the associated lentivirus for shRNA were prepared by Hanyin Lmt. Co. (Shanghai, China). To obtain cells with stable knockdown of HIF1a, cells were cultured in six-well plates at a density of $1 \times 10^{5}$ cells per well. On the following day, cells were infected with the same titre lentivirus with $8 \mu \mathrm{g} / \mathrm{ml}$ polybrene. Seventy-two $\mathrm{h}$ post viral infection, GFP-positive cells were detected by a fluorescence microscope, and the culture medium was charged with $4 \mu \mathrm{g} / \mathrm{ml}$ puromycin for selection for 14 days. The puromycin-resistant cells were amplified in medium containing $2 \mu \mathrm{g} / \mathrm{ml}$ puromycin for seven days and then transferred to a medium without puromycin. The clones were designated as KD or NC cells. Clones derived from Hif1a sh2 were designated as Hif1a KD, and clones from Wnt4 sh3 were designated as WNT4 KD.

\section{Scratch assay}

Cells $\left(1 \times 10^{6} /\right.$ well $)$ were cultured in 6-well plates, and the monolayers were scratched with 200- $\mu$ l pipette tips. The injured areas were photographed $0 \mathrm{~h}$ and $48 \mathrm{~h}$ later using a light microscope (CKX43, Olympus) at $100 \mathrm{x}$. The wound-closure percentages were calculated using the following formula: (1-[current wound size/initial wound size])*100. The assays were conducted in triplicate. 


\section{Transwell assay}

Cells $\left(1 \times 10^{4} /\right.$ well $)$ were suspended in serum-free medium and seeded in the upper chamber of Transwell inserts (Millipore, Billerica, MA) with a pore size of $8 \mu \mathrm{m}$. The lower chamber was charged with complete medium containing $10 \%$ FBS as a chemo-attractant. After $24 \mathrm{~h}$, cells on the upper surface of the insert were gently detached with a cotton swab. The invading cells were fixed with $4 \%$ paraformaldehyde for 20 mins (Sigma-Aldrich) and then stained with crystal violet. Five random microscopic fields were examined for each well using a light microscope (CKX43, Olympus) at 200x. All assays were performed in triplicate.

\section{Statistical analysis}

All statistical analyses were performed by SPSS v17.0 (Chicago, IL). All results were considered as significant at a $P$ value $<0.05$.

\section{Results}

\section{Exosomes derived from hypoxic CRC cells increased the migration and invasion ability of normoxic CRC cells.}

To investigate the functions of hypoxic CRC cell-derived exosomes in CRC progression, such exosomes were isolated from two different CRC cell lines. The morphology of the exosomes was observed via transmission electron microscopy (Fig. 1A). As shown in Fig. 1a, the diameter of the exosomes ranged from $30-100 \mathrm{~nm}$. Western blotting showed that the exosomes were enriched with the exosomal markers CD9a and CD63 (Fig. 1B), which indicated the effective isolation of the exosomes.

To determine the effects of hypoxic CRC cell-derived exosomes on normoxic CRC cells, we analysed the migration and invasion abilities of normoxic CRC cells treated with exosomes isolated from hypoxic CRC cells. Scratch assay results showed that hypoxic CRC cell-derived exosomes from both CRC cell lines significantly promoted normoxic CRC cell migration compared with exosomes isolated from normoxic CRC cells (Fig. 1C and 1D). In addition, Transwell assay results showed that hypoxic CRC cell-derived exosomes from both CRC cell lines enhanced the invasion ability of normoxic CRC cells relative to the normoxic control (Fig. 1E and 1F). These results demonstrate that hypoxic colorectal cancer cell-released exosomes promoted the migration and invasion of normoxic CRC cells.
A

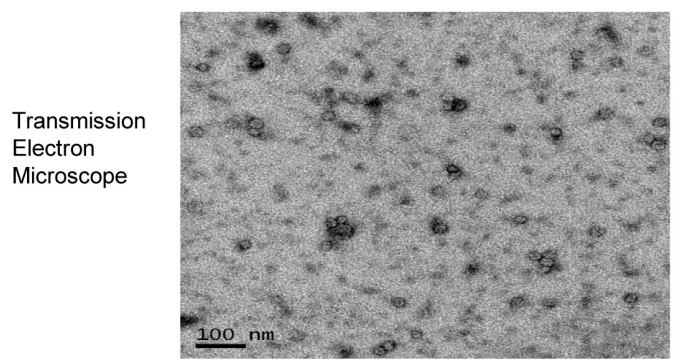

C

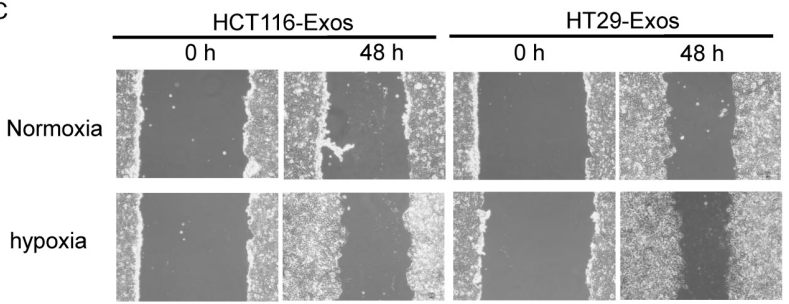

E
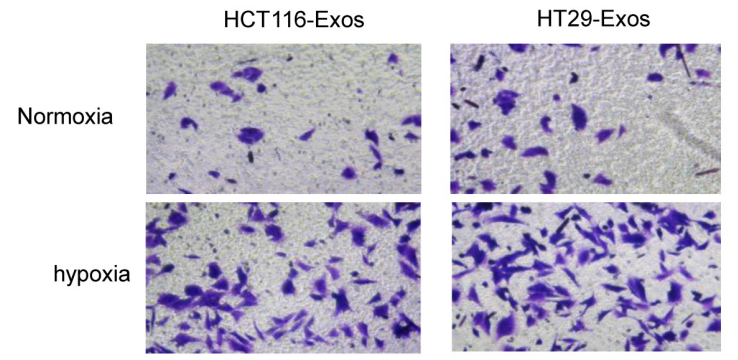

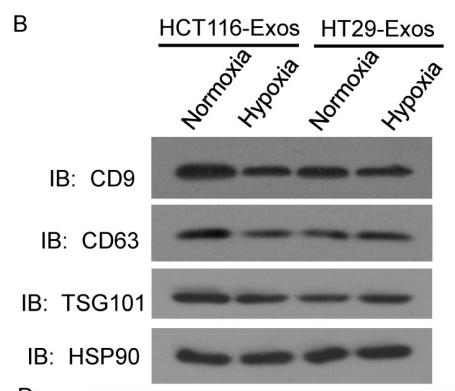

D

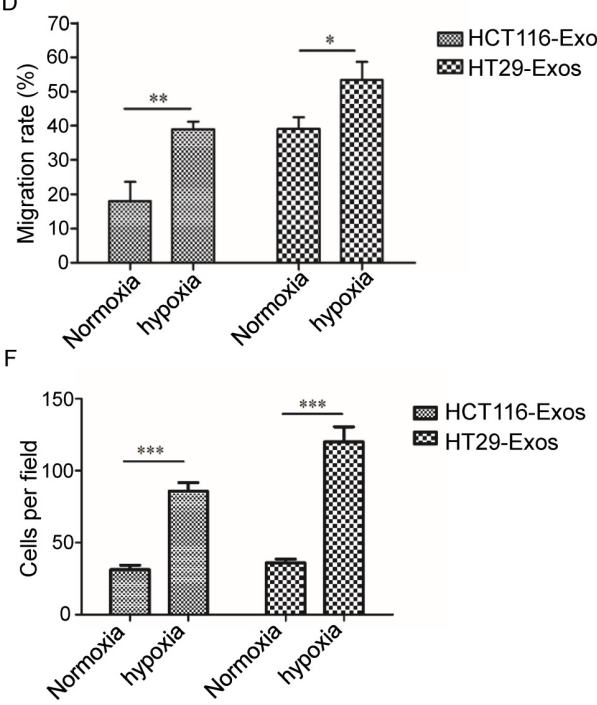

Figure 1. Exosomes derived from hypoxic CRC cells increased the migration and invasion ability of normoxic CRC cells. (A) Representative electron micrograph of exosomes isolated from hypoxic CRC cells revealing their typical morphology and size $(30-100 \mathrm{~nm})$. Scale bar, $100 \mathrm{~nm}$. (B) Western blot analysis showing the presence of CD9, CD63, TSG101 and HSP90 in exosomes derived from normoxic or hypoxic HT29 and HCT116 cells. (C, D) Scratch assay results showing that exosomes derived from hypoxic CRC cells increased the migration ability of normoxic CRC cells. (E, F) Transwell assay results showing that exosomes derived from hypoxic HT29 cells significantly increased the invasion ability of normoxic $C R C$ cells. ${ }^{* * *} P<0.001$ vs. normoxic group. ${ }^{* *} P<0.01$ vs. normoxic group; $* P<0.05$ vs. normoxic group. 
A

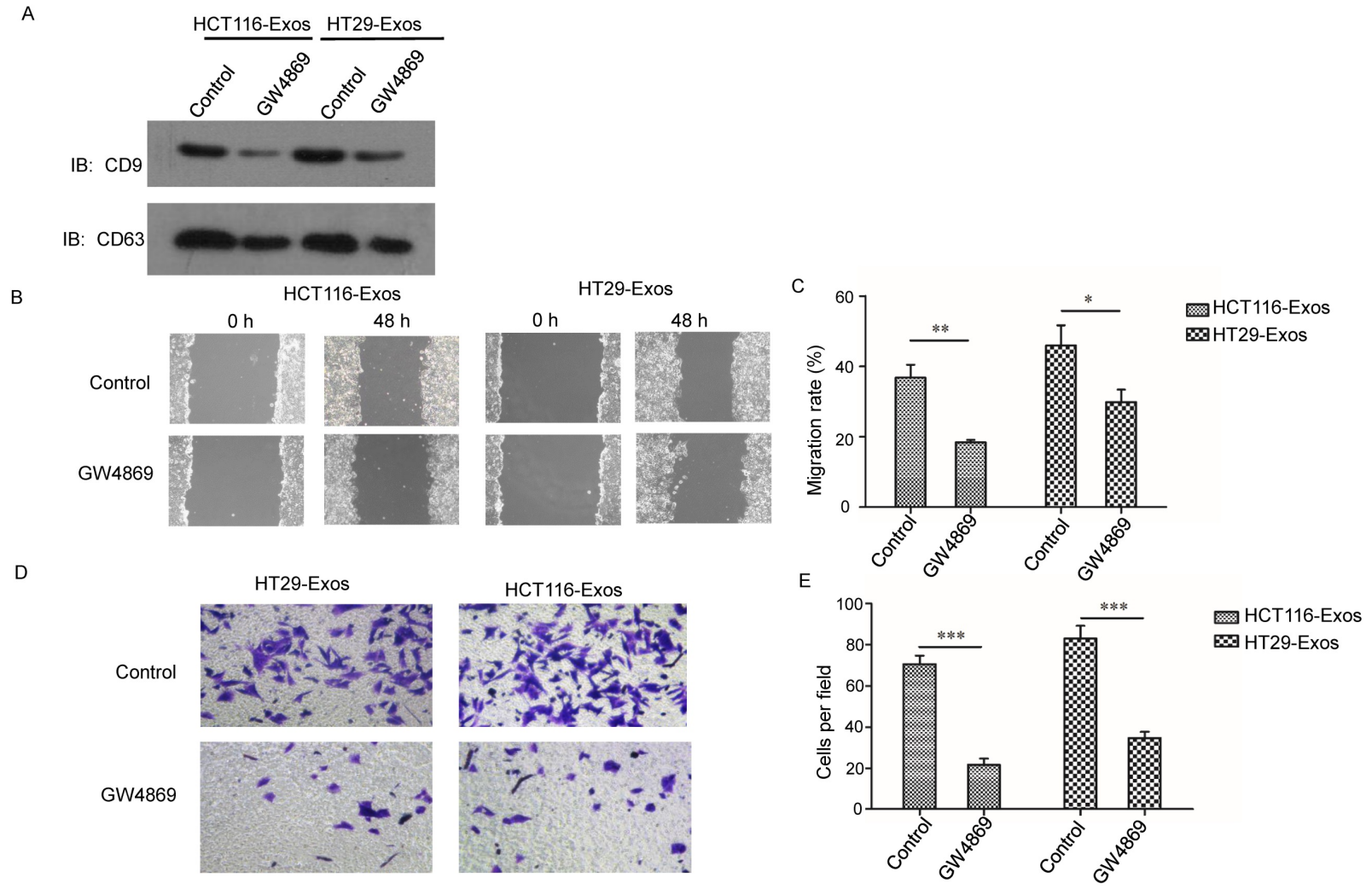

Figure 2. Suppression of exosome secretion reduced hypoxic exosome-induced migration and invasion of normoxic CRC cells. (A) Western blot analysis confirmed the efficient suppression of exosome release by GW4869 in HT29 and HCT116 cells. (B, C) Scratch assay results showing that exosomes derived from hypoxic HCT116 and HT29 cells with GW4869 treatment significantly reduced the migration ability of normoxic CRC cells compared with the control. (D, E) Transwell assay results showing that exosomes derived from hypoxic HCT1 16 and HT29 cells with GW4869 treatment significantly reduced the invasion ability of normoxic CRC cells compared with the control. ${ }^{* *} \mathrm{P}<0.001$ vs. control group; ${ }^{* * P}<0.01$ vs. control group; " $\mathrm{P}<0.05$ vs. control group.

\section{Suppression of exosome secretion reduced hypoxic exosome-induced migration and invasion of normoxic CRC cells.}

To further verify the effects of hypoxic CRC cell-derived exosomes on normoxic CRC cells, exosome generation was blocked with GW4869, an exosomal release inhibitor (23-24). As shown in Fig. 2A, exosome release was effectively suppressed after GW4869 treatment (Fig. 2A). Scratch assay results showed that GW4869 significantly reduced normoxic CRC cell migration induced by hypoxic CRC cell-derived exosomes (Fig. 2B and 2C). In addition, Transwell assay results showed that GW4869 treatment inhibited normoxic CRC cell invasion induced by hypoxic CRC cell-derived exosomes (Fig. 2D and 2E). These results further verified that hypoxic colorectal cancer cell-derived exosomes increased the movement and invasion of normoxic CRC cells.

\section{Hypoxic exosome-induced migration and invasion of normoxic CRC cells was dependent on HIFla.}

Considering that the exosomes used in this study were collected under hypoxic conditions, we examined the expression of HIF-1a, the most important hypoxia response factor, in the hypoxic
CRC cell lines. The results from both qRT-PCR and western blotting demonstrated that the expression of HIF-1 was increased in hypoxic cells (Fig. 3A and 3B). We further studied whether these phenomena were regulated by HIF-1a. We knocked down HIF-1a in both CRC cell lines (Fig. 3C and 3D). Scratch assay results showed that hypoxic exosomes collected from HIF-1a knockdown cells did not significantly increase normoxic CRC cell migration compared to control cells (Fig. 3E and 3F). Further, Transwell assay results showed that hypoxic exosomes collected from HIF-1a knockdown cells decreased normoxic CRC cell invasion compared to control cells (Fig. 3G and 3H). These results showed that hypoxic exosome-mediated migration and invasion of normoxic CRC cells was dependent on HIF1A.

\section{Exosomal Wnt4 facilitated hypoxic exosome-induced migration and invasion.}

The qRT-PCR results showed that compared to the normoxic control, the mRNA level of Wnt4 was significantly higher in hypoxic CRC (Fig. 4A). Furthermore, Western blotting showed that the protein levels of Wnt4 were higher in hypoxic exosomes than in exosomes derived from normoxic cells (Fig. 4B). HIF-1a knockdown reduced the Wnt4 mRNA levels in hypoxic exosomes (Fig. 4C). Western 
blotting further showed reduced Wnt4 protein levels in CRC cells cultured with hypoxic exosomes collected from HIF-1a knockdown cells (Fig. 4D).

We further investigated the effects of Wnt4 knockdown on CRC cell migration and invasion (Fig. 5A and 5B). Wnt4 knockdown in normoxic CRC cells abrogated hypoxic exosome-mediated migration enhancement (Fig. 5C and 5D). The results of the Matrigel-Transwell assay revealed that Wnt4 suppression reduced CRC cell invasion relative to the negative control (Fig. 5E and 5F). These results showed that exosomal Wnt4 mediated hypoxic exosome-induced migration and invasion.

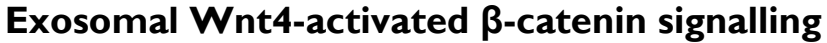 was required for migration and invasion of normoxic CRC cells.}

The Wnt/ $\beta$-catenin signalling pathway is one of the most important pathways in cancer; thus, we further examined $\beta$-catenin signalling in normoxic cells treated with hypoxic exosomes. Higher nuclear location of $\beta$-catenin was revealed in hypoxic exosome-treated normoxic CRC cells than in the control by Western blotting (Fig. 6A). To determine the functions of $\beta$-catenin in cells treated with hypoxic CRC cell-derived exosomes, we simultaneously treated normoxic cells with the $\beta$-catenin inhibitor ICG001. The scratch assay showed that the increased migration of normoxic cells induced by hypoxic CRC cell-derived exosomes was abolished by treatment with ICG001 (Fig. 6B and 6C). Transwell assay results also showed that ICG001 inhibited the invasion of normoxic cells induced by exosomes derived from hypoxic CRC cells (Fig. 6D and 6E). These results suggest that exosomal Wnt4-activated $\beta$-catenin signalling was required for migration and invasion of normoxic CRC cells.

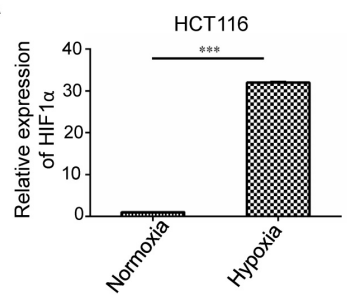

C
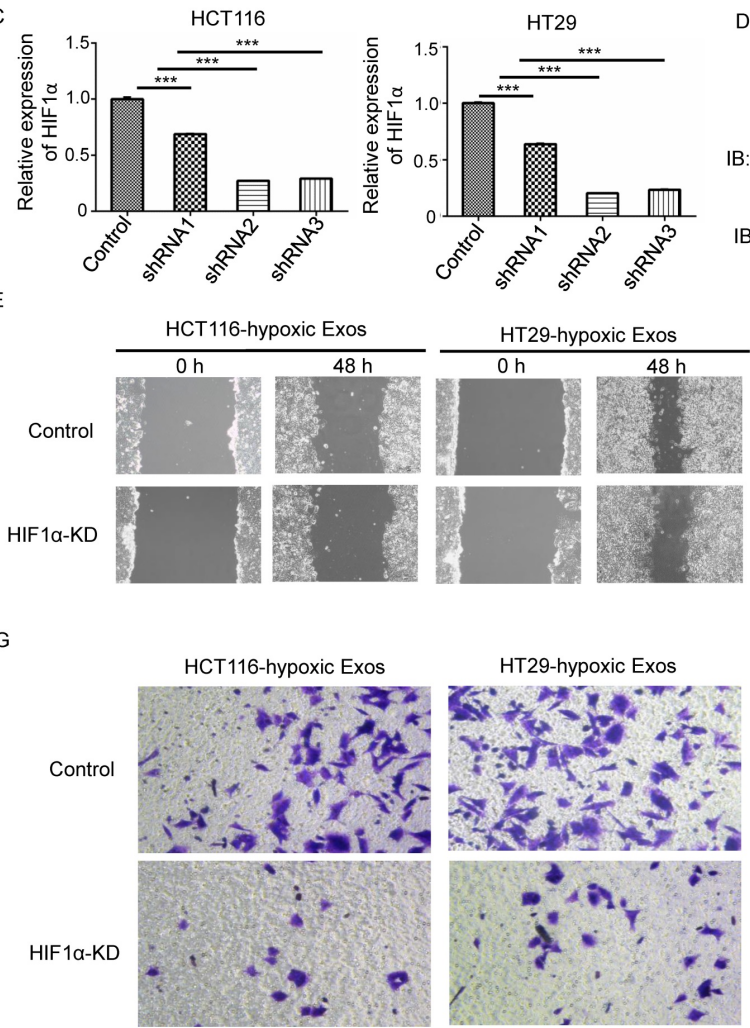

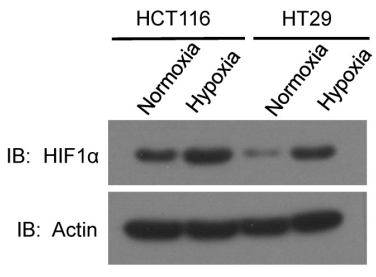

IB: HIF1a

IB: Actin

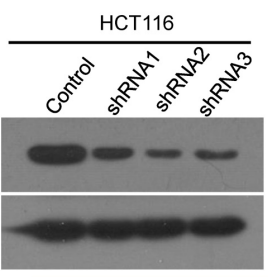

IB: HIF1a

IB: Actin

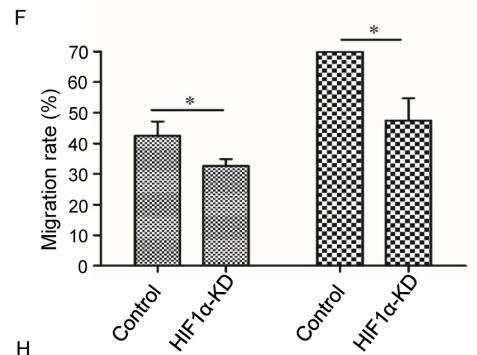

H

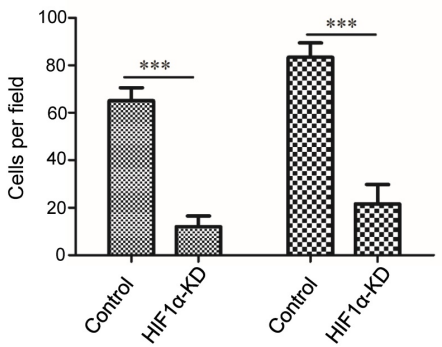

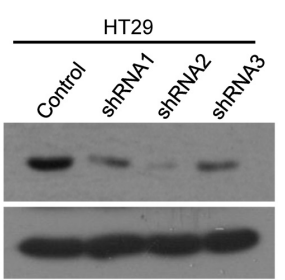

- HCT116-Exos HT29-Exos

Figure 3. Hypoxic exosome-induced migration and invasion ability of normoxic CRC cells was dependent on HIF1A. (A) Real-time PCR analysis of HIF1a expression in HCT116 and HT29 cells under hypoxia. (B) Western blot analysis of HIF1a expression in HCT116 and HT29 cells under hypoxia. (C) Real-time PCR analysis of HIF1a expression in HCT116 and HT29 cells with or without HIFla knockdown. (D) Western blot analysis showing HIF1a expression in HCT116 and HT29 cells with or without HIFla knockdown. (E, F) Scratch assay results showing that exosomes derived from hypoxic HCT116 and HT29 cells with HIF1 $\alpha$ knockdown significantly reduced the migration ability of normoxic CRC cells compared with the control. (G, H) Transwell assay results showing that exosomes derived from hypoxic HCT116 and HT29 cells with HIF1a knockdown significantly reduced the invasion ability of normoxic CRC cells compared with the control. ${ }^{* * * *} \mathrm{P}<0.001$ vs. control group; ${ }^{*} \mathrm{P}<0.05$ vs. control group. 


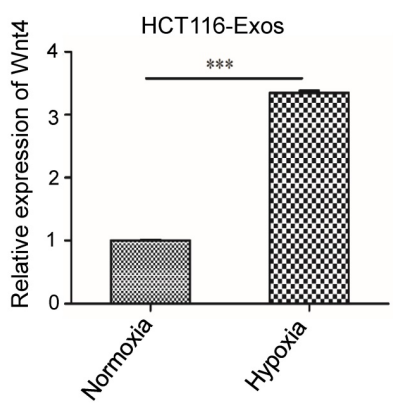

C

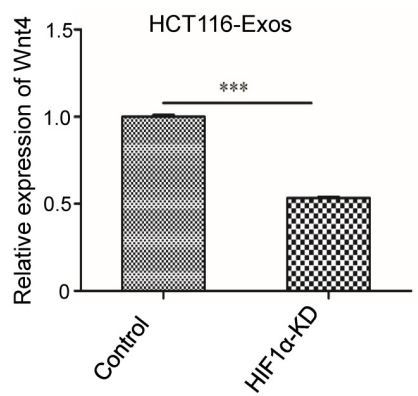

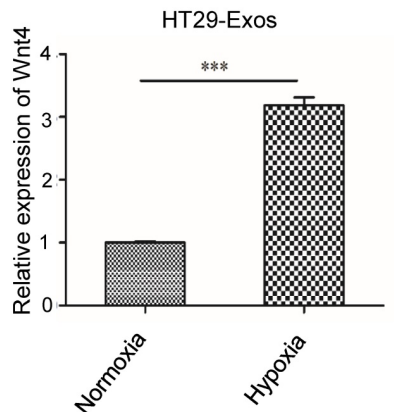

D

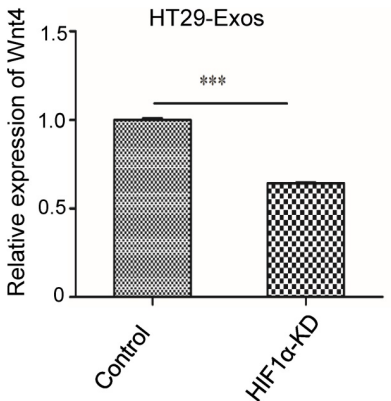

IB: Wnt4

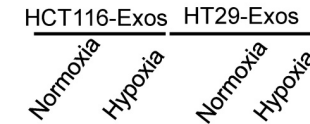

IB: Actin

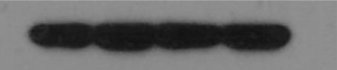

IB: Wnt4

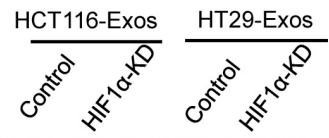

IB: Actin

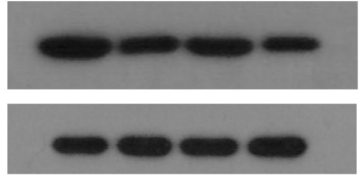

Figure 4. HIFla upregulated Wnt4 mRNA in hypoxic exosomes. (A) Real-time PCR analysis of Wnt4 in exosomes derived from HCT116 and HT29 cells under hypoxia. Exosomes derived from normoxic cells served as a control. (B) Western blot analysis showing that WNT4 protein levels increased in normoxic CRC cells after incubation with exosomes derived from hypoxic HT29 and HCT116 cells. (C) Real-time PCR analysis of Wnt4 in hypoxic exosomes derived from HCT1 16 and HT29 cells with HIF1a knockdown. (D) Western blot analysis of Wnt4 in normoxic CRC cells cultured with hypoxic exosomes derived from HCT116 and HT29 cells with HIFla knockdown. Pso $<0.001$ vs. control group.

A

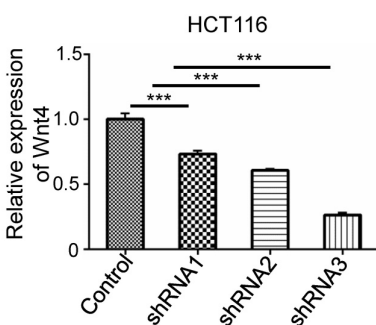

C

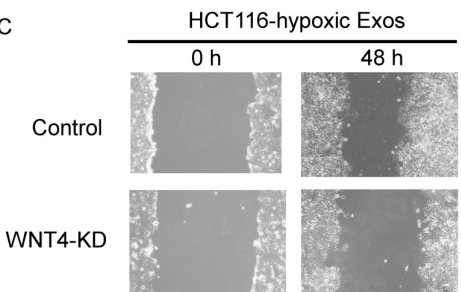

$\mathrm{E}$

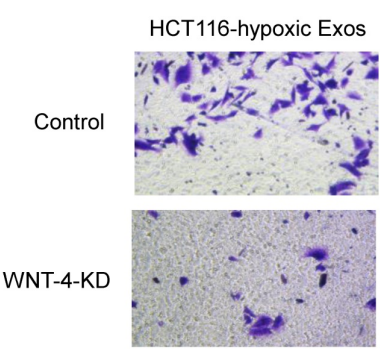

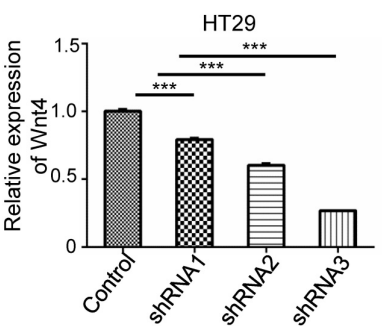

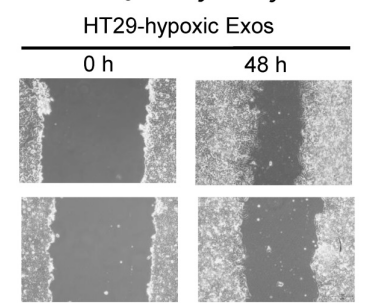

HT29-hypoxic Exos

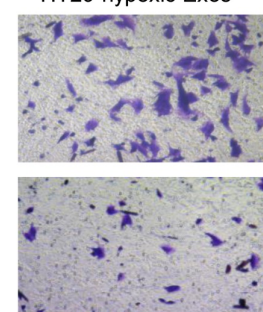

IB: Wnt4

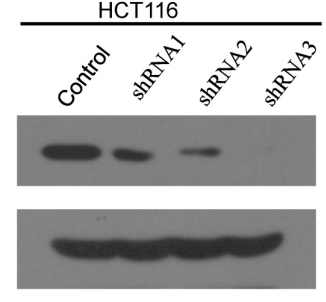

IB: Wnt 4

IB: Actin

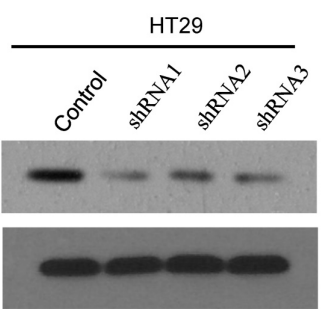

D

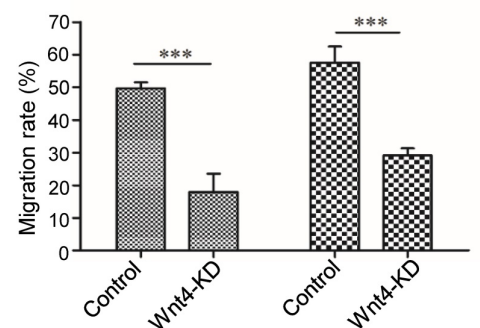

$\mathrm{F}$

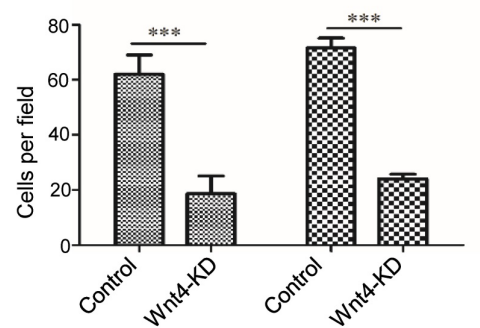

Figure 5. Exosomal Wnt4 mediated hypoxic exosome-induced migration and invasion. (A) RT-PCR analysis of Wnt4 in HCT116 and HT29 cells with or without Wnt4 knockdown. (B) Western blot analysis of Wnt4 in HCT116 and HT29 cells with or without Wnt4 knockdown. (C, D) Scratch assay results showing that Wnt4 knockdown in normoxic CRC cells reduced the hypoxic exosome-induced migration ability of normoxic CRC cells compared with the control. (E, F) Transwell assay results showing that Wnt4 knockdown in normoxic CRC cells reduced the hypoxic exosome-induced invasion ability of normoxic CRC cells compared with the control. ${ }^{* * *} P<0.001$ vs. control group. 
A

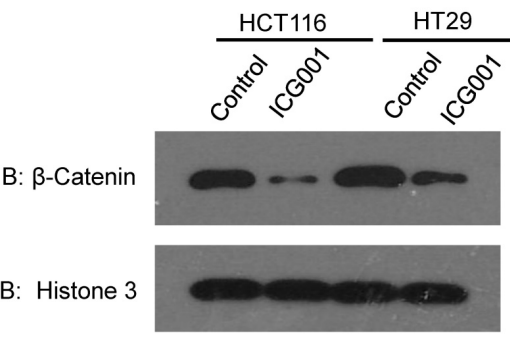

B

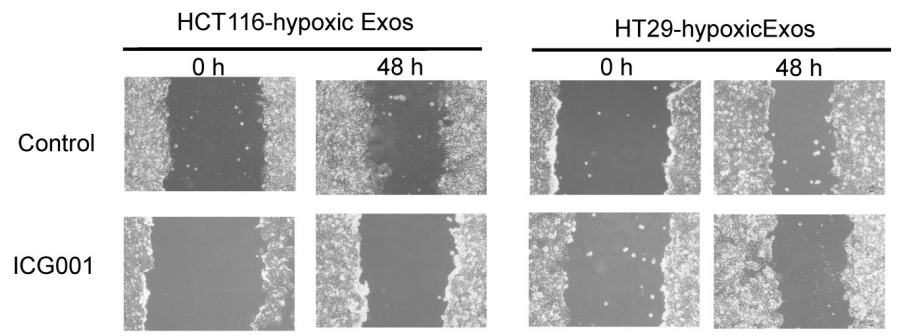

C
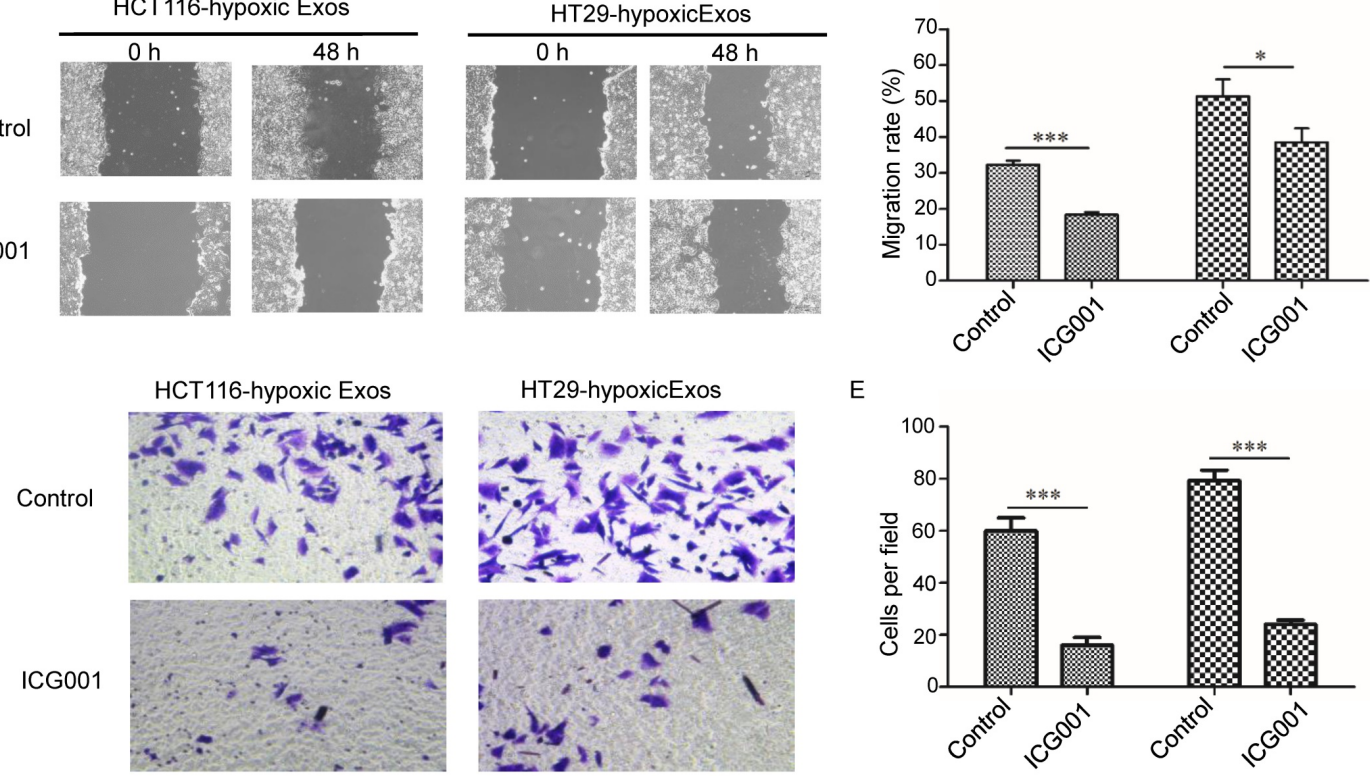

HCT116-Exos

$\$$ HT29-Exos

E

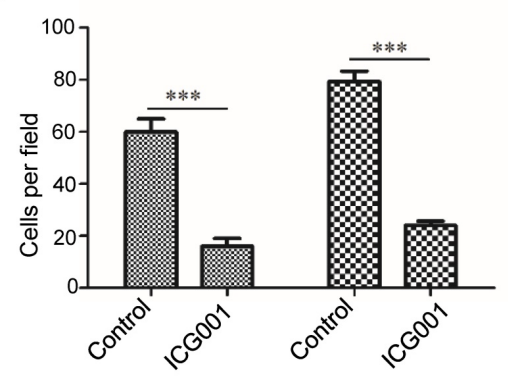

HCT116-Exos

$\infty$ HT29-Exos

Figure 6. Exosomal Wnt4 activated $\beta$-catenin signalling was required for exosome-induced migration and invasion. (A) Western blot analysis showing that nuclear $\beta$-catenin was increased in normoxic cells treated with exosomes derived from hypoxic HT29 and HCT116 cells. (B, C) Scratch assay results showing that normoxic cells treated with the $\beta$-catenin signalling inhibitor ICG001 exhibited significantly reduced migration in the presence of hypoxic exosomes. (D, E) Transwell assay results showing that normoxic cells treated with the $\beta$-catenin signalling inhibitor ICG001 exhibited significantly reduced invasion in the presence of hypoxic exosomes. group; $* \mathrm{P}<0.05$ vs. control group.

\section{Discussion}

Recent studies have shown that exosomes facilitate cell-cell communication by transferring RNA and protein between cells. As a result, exosomes play significant roles in a variety of physiological and pathological processes including cancer (25-28). In this study, we investigated the functions and associated pathways of hypoxic CRC cell-derived exosomes. We found that exosomes released by hypoxic colorectal cancer cells promote the metastasis of normoxic CRC cells through Wnt4-induced $\beta$-catenin signalling. This is the first study to reveal that exosomes mediate $\mathrm{Wnt} / \beta$-catenin signalling in normoxic cells by hypoxic CRC cells.

Hypoxia has been found to be an important element for tumour progression through the regulation of signalling pathways participating in various processes, such as angiogenesis, invasion, metabolism and genetic instability $(29,30)$. HIF1a is a central factor in hypoxia. In cancer cells, hypoxia stabilizes HIF1a, which leads to the expression of genes regulating survival, tumour vascularization and metastasis (31-33). Though recent studies have identified the functions of exosomes in tumour progression, the cellular functions and underlying mechanisms of hypoxic exosomes have not been well clarified. Exosomes released from hypoxic oral squamous cell carcinoma cells (34), glioma cells (35) and leukaemia cells (28) have been found to promote tumour progression by regulating phenotypic modulation of endothelial cells or normoxic tumour cells. We previously found that exosomes released by hypoxic CRC cells stimulated the proliferation and migration of endothelial cells. Inhibition of exosomal secretion via RAB27A knockdown in cancer cells significantly reduced exosome-mediated proliferation and migration of endothelial cells (13). Consistent with the findings of previous reports, our results indicate that exosomes are key transducers in CRC metastasis.

Exosomes are known to contain mRNAs, non-coding RNAs, and proteins. The horizontal transfer of mRNAs has been found in cancer $(8,11)$. In this study, we found that Wnt4 mRNA was transferred from hypoxic CRC cells via exosomes. 
Accumulating evidence supports the importance of the Wnt signalling pathway in development and in diseases such as cancer. For example, Julia et al. demonstrated that Wnts are transported through endosomal compartments into exosomes (36). Bing et al. showed that exosomes released by huMSC contain Wnt4 protein, which prompts wound healing (37). In this study, we found that Wnt4 mRNAs were enriched in exosomes instead of Wnt4 protein, which provides evidence for another mechanism of exosome-mediated Wnt transfer. Furthermore, we determined that exosomal Wnt4 was upregulated by HIF1a, which is consistent with previous reports that describe the influence of HIF-1a and HIF-2a expression on circulating exosomes (38).

The Wnt/catenin signalling pathway is a classic pathway in cancer (16). Without Wnt signalling, $\beta$-catenin is degraded in the cytoplasm, while with Wnt signalling, $\beta$-catenin accumulates in the cytoplasm and translocates into the nucleus, where it functions as a transcriptional cofactor (17). Given this pattern, we examined $\beta$-catenin signal activation in endothelial cells and found that hypoxic exosomes activate this signalling pathway. This signalling might be at least partially mediated by Wnt4. As the exosomal contents are very complex, further investigation is needed to determine whether additional factors are involved in regulating the activity of the Wnt/ $\beta$-catenin signalling pathway in hypoxic CRC cell-derived exosomes.

In summary, we found that hypoxic CRC cell-derived exosomes promote tumour progression through delivery of Wnt 4 mRNA to normoxic CRC cells and result in $\beta$-catenin activation in those cells. Furthermore, exosomes derived from hypoxic CRC cells facilitate normoxic CRC cell migration and invasion. Our findings provide new implications for cancer therapies involving exosome inhibition.

\section{Acknowledgements}

This study was supported by the Shenyang Science and Technology Plan project (no. 18-014-4-31).

\section{Competing Interests}

The authors have declared that no competing interest exists.

\section{References}

1. Jemal A, Bray F, Center MM, et al. Global cancer statistics. CA Cancer J Clin. 2011; 61: 69-90.

2. Levine JS, Ahnen DJ. Clinical practice. Adenomatous polyps of the colon. N Engl J Med. 2006; 355: 2551-7.

3. Ciombor KK, Wu C, Goldberg RM. Recent therapeutic advances in the treatment of colorectal cancer. Annu Rev Med. 2015; 66:83-95.

4. Mo S, Cai G. Multidisciplinary Treatment for Colorectal Peritoneal Metastases: Review of the Literature. Gastroenterol Res Pract. 2016;2:1-8.

5. Havelund BM, Holdgaard PC, Rafaelsen SR, et al. Tumour hypoxia imaging with 18 F-fluoroazomycinarabinofuranoside PET/CT in patients with locally advanced rectal cancer. Nucl Med Commun. 2013;34(2):155-61.

6. Rasheed S, Harris AL, Tekkis PP, et al. Hypoxia-inducible factor-1alpha and -2alpha are expressed in most rectal cancers but only hypoxia-inducible factor-1alpha is associated with prognosis. $\mathrm{Br} \mathrm{J}$ Cancer. 2009;100(10):1666-73.

7. Thery C, Zitvogel L, Amigorena S. Exosomes: composition, biogenesis and function. Nat Rev Immunol. 2002;2:569-79.

8. Mathivanan S, Ji H, Simpson RJ. Exosomes: extracellular organelles important in intercellular communication. J Proteomics. 2010;73:1907-20.

9. El AS, Mager I, Breakefield XO, et al. Extracellular vesicles: biology and emerging therapeutic opportunities. Nat Rev Drug Discov. 2013;12:347-57.

10. Yamada N, Kuranaga Y, Kumazaki M, et al. Colorectal cancer cell-derived extracellular vesicles induce phenotypic alteration of T cells into tumor-growth supporting cells with transforming growth factor- $\beta 1$-mediated suppression. Oncotarget. 2016; 7(19): 27033-43.

11. Ji H, Greening DW, Barnes TW, et al. Proteome profiling of exosomes derived from human primary and metastatic colorectal cancer cells reveal differential expression of key metastatic factors and signal transduction components. Proteomics. 2013; 13(10-11):1672-86.

12. Yoon-Keun K, Dong-Sic C, Ji K, et al. Colorectal cancer cell-derived microvesicles are enriched in cell cycle-related mRNAs that promote proliferation of endothelial cells. BMC Genomics. 2009;10(1):556-556.

13. Huang $Z$, Feng Y. Exosomes derived from hypoxic colorectal cancer cells promotes angiogenesis through Wnt4 induced $\beta$-catenin signaling in endothelial cells. Oncol Res. 2017; 25(5):651-61.

14. Clevers H. Wnt/ beta-catenin signaling in development and disease. Cell. 2006;127:469-80.

15. Gregorieff A, Clevers H. Wnt signaling in the intestinal epithelium: from endoderm to cancer. Genes Dev. 2005;19:877-90.

16. Reya T, Clevers H. Wnt signalling in stem cells and cancer. Nature. 2005;434:843-50.

17. MacDonald BT, Tamai $\mathrm{K}, \mathrm{He} X$. Wnt/beta-catenin signaling: components, mechanisms, and diseases. Dev Cell. 2009;17:9-26.

18. Li $\mathrm{W}$, Zhang $\mathrm{Y}$, Zhang $\mathrm{M}$, et al. Wnt4 is overexpressed in human pituitary adenomas and is associated with tumor invasion. J Clin Neurosci. 2014;21(1):137-41.

19. Miyakoshi T, Takei M, Kajiya H, et al. Expression of Wnt4 in human pituitary adenomas regulates activation of the beta-catenin-independent pathway. Endocr Pathol. 2008;19(4):261-73.

20. Brisken C, Hess K, Jeitziner R. Progesterone and Overlooked Endocrine Pathways in Breast Cancer Pathogenesis. Endocrinology. 2015;156(10):3442-50.

21. Zhu Y, Zhang B, Gong A, et al. Anti-cancer drug 3,3'-diindolylmethane activates Wnt4 signaling to enhance gastric cancer cell stemness and tumorigenesis. Oncotarget. 2016;7(13):16311-24.

22. Goldberg MA, Dunning SP, Bunn HF. Regulation of the erythropoietin gene: evidence that the oxygen sensor is a heme protein. Science. 1988;242(4884):1412-5.

23. Richards KE, Zeleniak AE, Fishel ML, et al. Cancer-associated fibroblast exosomes regulate survival and proliferation of pancreatic cancer cells. Oncogene. 2016;36(13):1770.

24. Ohshima K, Kanto K, Hatakeyama $\mathrm{K}$, et al. Exosome-mediated extracellular release of polyadenylate-binding protein 1 in human metastatic duodenal cancer cells. Proteomics. 2014;14(20):2297-306.

25. van Niel G, Porto-Carreiro I, Simoes S, et al. Exosomes: a common pathway for a specialized function. J Biochem. 2006;140:13-21.

26. Peinado $\mathrm{H}$, Aleckovic $M$, Lavotshkin $S$, et al. Melanoma exosomes educate bone marrow progenitor cells toward a pro-metastatic phenotype through MET. Nat Med. 2012;18:883-91.

27. Webber J, Steadman R, Mason MD, et al. Cancer exosomes trigger fibroblast to myofibroblast differentiation. Cancer Res. 2010;70: 9621-30.

28. Tadokoro H, Umezu T, Ohyashiki K, et al. Exosomes derived from hypoxic leukemia cells enhance tube formation in endothelial cells. J Biol Chem. 2013;288:34343-51.

29. Brahimi-Horn MC, Chiche J, Pouyssegur J. Hypoxia and cancer. J Mol Med. 2007;85:1301-7.

30. Lu X, Kang Y. Hypoxia and hypoxia-inducible factors: master regulators of metastasis. Clin Cancer Res. 2010;16:5928-35.

31. Nagaraju GP, Bramhachari PV, Raghu G, et al. Hypoxia inducible factor-1a: Its role in colorectal carcinogenesis and metastasis. Cancer Lett. 2015;366(1):11-8.

32. Joseph JV, Conroy S, Pavlov $\mathrm{K}$, et al. Hypoxia enhances migration and invasion in glioblastoma by promoting a mesenchymal shift mediated by the HIF1a-ZEB1 axis. Cancer Lett. 2015;359(1):107-16.

33. Zhang L, Huang G, Li X, et al. Hypoxia induces epithelial-mesenchymal transition via activation of SNAI1 by hypoxia-inducible factor -1a in hepatocellular carcinoma. BMC Cancer. 2013;13:108. 
34. Li L, Li C, Wang S, et al. Exosomes Derived from Hypoxic Oral Squamous Cell Carcinoma Cells Deliver miR-21 to Normoxic Cells to Elicit a Prometastatic Phenotype. Cancer Res. 2016;76(7):1770-80.

35. Kucharzewska P, Christianson HC, Welch JE, et al. Exosomes reflect the hypoxic status of glioma cells and mediate hypoxia-dependent activation of vascular cells during tumor development. Proc Natl Acad Sci U S A. 2013;110:7312-7.

36. Gross JC, Chaudhary V, Bartscherer K, et al. Active Wnt proteins are secreted on exosomes. Nat Cell Biol. 2012;14(10):1036-45.

37. Menck K, Klemm F, Gross JC, et al. Induction and transport of Wnt 5a during macrophage-induced malignant invasion is mediated by two types of extracellular vesicles. Oncotarget. 2013;4(11):2057-66.

38. Zhang B, Wang M, Gong A, et al. HucMSC-exosome mediated-Wnt4 signaling is required for cutaneous wound healing. Stem Cells. 2015;33(7):2158-68 